\title{
Radar Corner Reflectors for Linear or Circular Polarization
}

\author{
G. Latmiral and A. Sposito \\ Contribution from the Istituto Universitario Navale, Naples, Italy
}

(Received August 2, 1961)

\begin{abstract}
When a grid of parallel wires is put in front of a plane or corner reflector, interesting effects of polarization conversion can be obtained. These effects may be used to construct trihedral corner reflectors which, contrary to the normal ones, are radarvisible even with circular polarization.
\end{abstract}

\section{Introductory Remarks}

It is known that echoes from rain become very attenuated with circular-polarized radar. Circular polarization can be obtained, e.g., by placing in front of the antenna of a horizontally-polarized radar the microwave equivalent in optics of a quarterwave birefracting plate. This consists of a set of metal strips inclined at an angle of $45^{\circ}$, of such dimensions that the component of the electrical field which is parallel to the strips is accelerated by $1 / 4$ period relative to the normal component. Upon leaving the polarizer, the two components differ in phase by a $1 / 4$ period instead of being in phase.

If a circular-polarized wave is incident upon a "nondepolarizing" target which reflects circular polarization without modifying it, the inverted "circular" wave is changed to "linear," at $90^{\circ}$ to the initial direction, upon passing through the " $\lambda / 4$ plate." The term "without modifying" does not deny the existence of an inversion in the direction of rotation relative to the propagation vector, which always happens in a single plane reflection or, generally, in odd-numbered plane reflections. One feature of this system is that the reflected wave does not pass into the waveguide feed of the antenna, nor does it activate the receiver. It thus follows that a circular-polarized radar echo from a sphere (e.g., a raindrop), a Luneberg lens-reflector or a plane or trihedral corner reflector becomes completely, or at least practically, invisible. Actually, a circularly polarized radar is rigorously blind only for targets which are symmetrical with respect to the radartarget line of sight. For such targets, the two "orthogonal null polarizations" [Copeland, 1960] are the two circular ones. However, the circularpolarized radar echo from a dihedral reflector becomes, instead, perfectly visible. It is because of double reflection, as well as depolarizing targets, that the echo from a ship becomes sufficiently visible even with circular polarization with an attenuation of only some db units, compared to linearly-polarized

${ }_{1}^{1}$ This research has been sponsored by the Rome Air Development Center of the Air Research and Development Command, through its European Office. signals. Under these conditions the echo from rain is attenuated by at least $20 \mathrm{db}$.

Dihedral corner reflectors are, however, less practical than the trihedral, and their echo is very unstable when the situation involves rolling buoys or vessels.

\section{Statement of Problem}

The problem can be proposed in this manner: Radar with circular polarization is almost completely free of echo due to rain. The latter, specially in the $3-\mathrm{cm}$ range, can mask signals from desired targets and create very hazardous situations. Unfortunately, with circular polarization, trihedral corner reflectors (and Luneberg lenses) are ineffective and this may cause grave risks particularly in cloudy areas where such corner reflectors are indispensable to render radarvisible such targets as small vessels on fire, barriers, and the like which are otherwise incapable of sufficient radar response.

What can be done to remedy such a situation? Obviously, if the solution should involve a corner reflector, the solution could not amount to more than modifying the polarization of the reflected wave, and adapting it suitably to radar use.

This objective can be better achieved instrumentally with an arrangement whereby the incident wave is not depolarized by absorption but by a true and appropriate conversion of polarization. Two types of converters can be considered in this connection:

\section{(A) $\lambda / 4$-Converter}

A converter which would be the equivalent in optics of a $\lambda / 8$ birefracting plate over a reflecting surface. The accelerated component ("extraordinary component vibrating parallel to the optical axis) "gains" an eighth of a wavelength outgoing, and another eighth upon return, totaling $\lambda / 4$. A conversion of polarization takes place from circular to linear (at $45^{\circ}$ relative to the optical axis) or inversely from linear (at $45^{\circ}$ to the optical axis) to circular. $^{2}$ The microwave strip polarizers show the

2 The sense of the circular polarization is that which superposes the vector of the accelerated component upon the vector of the nonaccelerated one. 
"extraordinary" vibration as being parallel to the strips.

We shall show how ordinary wires rather than strips can be used in a very simple and practical manner for the same purpose. Wire grid polarizers are essentially reflectors, whereas strip polarizers operate either by reflection or by "transparency." In the latter case, however, the efficiency of conversion is generally minor because of reflection losses.

\section{(B) $\lambda / 2$ - Converter}

A converter which would be the equivalent of a $\lambda / 4$ birefracting plate, set over a reflecting mirror. Between incidence and emergence, an effect is obtained equivalent to that of a $\lambda / 2$ plate: rotation by $90^{\circ}$ of the electric vector. In other words, when the optical axis is inclined $45^{\circ}$ relative to the vertical, there is conversion of polarization from horizontal to vertical and vice versa, or from right circular to left circular and vice versa. The same considerations hold for strips (or wires).

How (A) and (B) function will now be considered.

$\left(\mathrm{A}^{\prime}\right)$. In the case of $(\mathrm{A})$, when the optical axis, or the direction of the strips or wires is $45^{\circ}$ from the vertical, radarvisibility is achieved equally with linear (horizontal or vertical) or circular polarization, but always with a loss of $3 \mathrm{db}$ in maximum echo.

If polarization is initially linear, reflection converts it into circular. There is a loss of $3 \mathrm{db}$ because the energy is divided equally between two orthogonal vibrations (with a phase difference of $\pi / 2$ ) only one of which is received.

If polarization is initially circular, it becomes linear horizontal or vertical, depending upon the sense of rotation and if there is a $\pm 45^{\circ}$ inclination of the optical axis from the vertical. If the direction of rotation coincides with the direction of inclination of the optical axis, the resulting polarization is vertical; otherwise, it is horizontal. In every case, however, there is a loss of $3 \mathrm{db}$. While the wave crosses the antenna polarizer, it is converted to circular, and half the signal power is lost at the end of the reception with a dipole.

$\left(\mathrm{B}^{\prime}\right)$. A converter of type (B) becomes perfectly radarvisible with circular polarization (as a smooth plane would be with linear) but altogether nonvisible with linear polarization because of the $90^{\circ}$ rotation due to polarization.

\section{Polarization Converters Operating With Wire Grid in Front of Reflectors-Plans and Calculations}

Polarization converters of types (A) and (B) mentioned in the preceding section and working with reflectors can be made to operate with parallel wire grids placed in front of plane, cylindrical and angular reflectors.

The possibility of operating reflection converters with the help of parallel wire grids has already been studied for some time. ${ }^{3}$ It is evident on the other

${ }^{3}$ We are indebted to Dr. Jean Ortusi of the General Wireless Telegraphy Co. (Compagnie Générale de Télégraphie Sans Fil) for calling our attention to Patent Notice No. 668231, "Reflektoranordnung "(Reflection Devices), by Mr. Julius Pintsch. communicated May 5, 1935, and published November 3, 1938. hand that whatever scheme is suitable to generate anisotropy and, particularly, birefraction (in the sense of a different response to vibrations parallel to a certain axis or normal to it) should give rise to a modification of polarization.

However, precise methods do not appear to be given for obtaining correct operation of the converter, nor such are adopted which serve the specific purpose of making corner reflectors radarvisible in circular polarization. However, modern technical literature gives us the necessary elements to make calculations for the converters. For example, MacFarlane [1946] and Wait [1954a] have derived formulas for the surface impedance of a grid (assumed indefinitely extended) of parallel wires, at $E$ paralle] and general incidence, respectively. Such a grid is indicated in figure 1.

When $d \leq \lambda_{0} / 2$ and the incident electric vector is parallel to the wires, the grid, for any angle of incidence, does not cause loss of energy due to sidewaves (which always appear paired symmetrically relative to the plane of the grid) but only the loss due to wave reflection or due to transmission without deviation.

The surface reactance of the grid, valid for $E$ parallel incidence, is

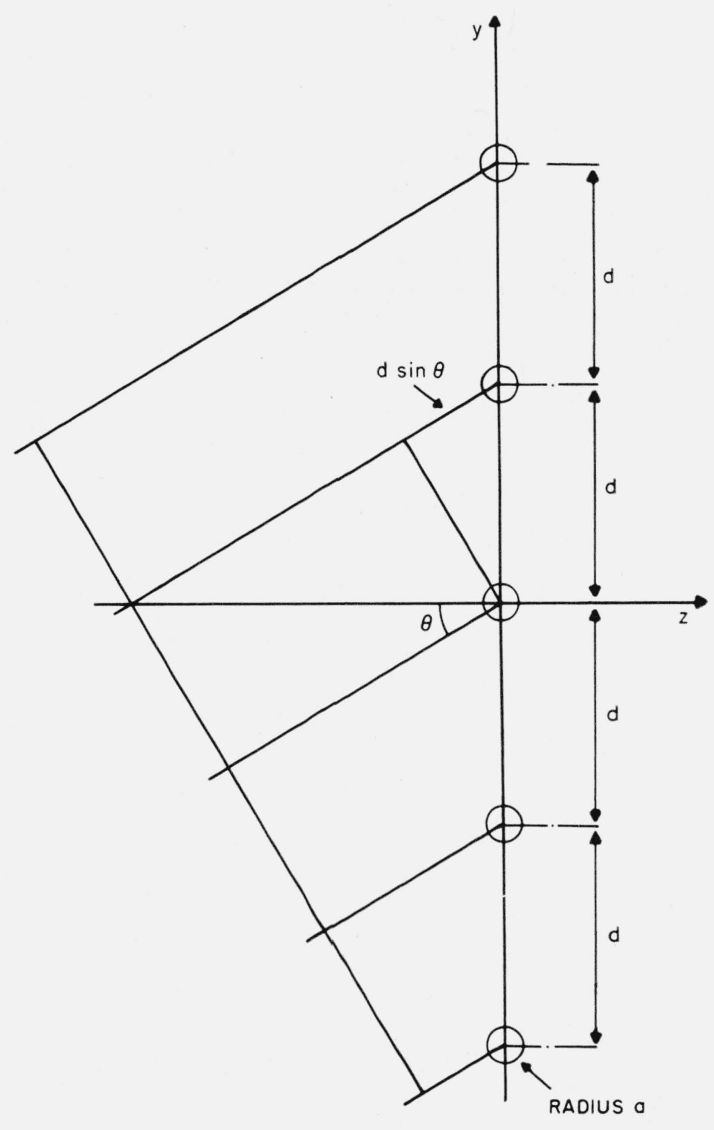

Figure 1. Parallel wire grid of indefinite extent. 


$$
\begin{aligned}
& X=\frac{Z_{0} d}{\lambda_{0}}\left[\ln \frac{d}{2 \pi a}+F\left(d / \lambda_{0}, \theta\right)\right] \\
& X \cong \frac{Z_{0} d}{\lambda_{0}} \ln \frac{d}{2 \pi a} \text { for } \quad a<<d<<\lambda_{0}
\end{aligned}
$$

where $Z_{0}=\sqrt{\frac{\mu_{0}}{\epsilon_{0}}}=120 \pi$ ohms, and where $d$ is the distance between wires; $a$, the radius of the wires; $\theta$, the angle of incidence, and $F\left(d / \lambda_{0}, \theta\right)$ is a function plotted in figure 2 .

For $a=0.5 \mathrm{~mm} ; \lambda_{0}=32 \mathrm{~mm} ; d=10 \mathrm{~mm}$; then $X \cong 143$ ohms. These values were adopted for the grid used in the experiment. The grid measured about $1 \mathrm{~m}^{2}$ and the wires ended on a metallic frame. The frame and a $20-\mathrm{cm}$ wide peripherical strip of the grid were screened by means of a 20 -db absorber. Owing to the skin effect, at a distance of a few tenths of centimeters from its end a relatively thick wire behaves at the $3-\mathrm{cm}$ band as if it were of infinite length. When a wave is incident upon the grid, and the electrical field is parallel to the wires, a reflection takes place at the grid plane. (If the field is normal to the wires and $a<<d<<\lambda_{0}$ the effect of the grid can be neglected.) The reflection and the transmission coefficients can be calculated according to the customary formulas, which have been experimentally verified by Decker [1959]

$$
r_{E}=\frac{Z_{2}-Z_{1}}{Z_{1}+Z_{2}} ; 1+r_{E}=t_{E}
$$

$Z_{1}$ is the wave impedance of the incident plane wave $\left(Z_{1}=Z_{0} \cdot \sec \theta\right)$ and $Z_{2}$ results from the parallel combination of $j X$ with the $Z_{0} \cdot \sec \theta$ relative to the vacuum ("extending" beyond the grid). In this case, one finds that

$$
r_{E}=\frac{-1}{1+j 2 X / Z_{0} \sec \theta} .
$$

If, however, there is a reflecting surface behind the grid pa,allel to the plane of the latter, at distance $l$ from it, ${ }^{4} Z_{2}$ is due to the parallel combination of $X$ with $j Z_{0} \operatorname{tg} \frac{2 \pi l}{\lambda_{0}}=j Z_{0} \alpha$ [Wait, 1954b]. The case of a plane wave with vector $E$ parallel (case $\mathrm{P}$ ) or normal (case $N$ ) to the wires, is shown in figure $3 \mathrm{P}$ and $3 \mathrm{~N}$, based on the known analogy between plane wave equations and the equation of propagation along the wires (principal mode).

If the incidence angle $\theta$ does not exceed $10^{\circ}$, one may in every case, with an approximation better than \pm 1.5 percent, consider the wave impedance of the incident and transmitted plane wave to be equal to $Z_{0}$. Given $r_{p}$, the reflection coefficient of wave $P(E$ parallel $) ; r_{n}$, the reflection coefficient of wave $N(E$ normal $) ;$ and $\phi_{p}$ and $\phi_{n}$ on the relative phases, then one can easily calculate $\left(\alpha=\operatorname{tg} \frac{2 \pi l}{\lambda_{0}}\right)$

i $l$ must be sufficiently large in comparison ${ }^{-}$with $d$ so as to make negligible the effect of the surface (or evanescent) waves which are confined to the immediate neighborhood of the grid [Wait, 1954a, eq 13]. The modification to the grid surface reactance when $l$ is not large, has also been worked out [Wait, 1954b].

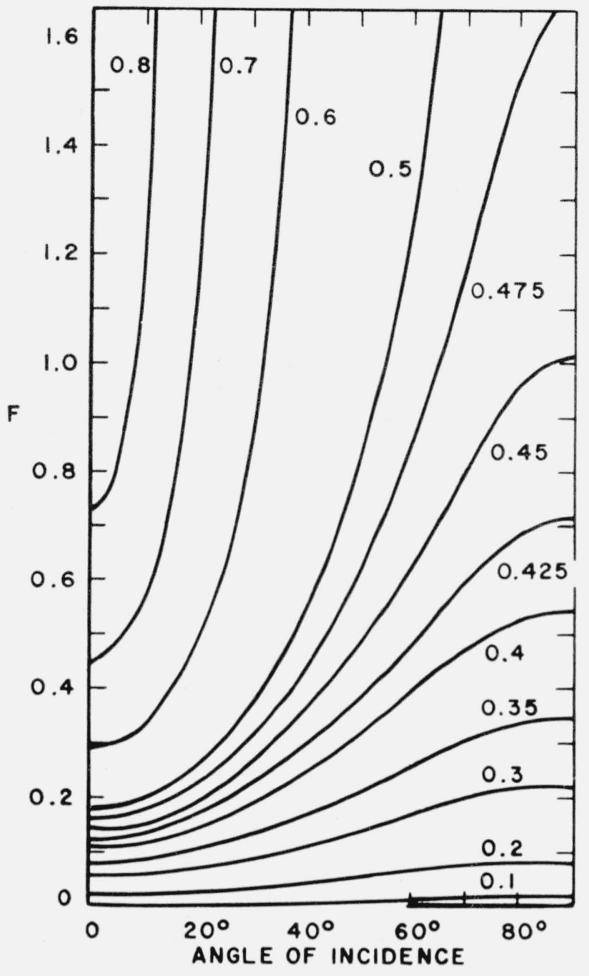

Figure 2. Correction term $\mathrm{F}\left(\mathrm{d} / \lambda_{0}, \theta\right)$ is a function of $\theta$ and the parameter $\mathrm{d} / \lambda_{0}$.

The graph is from G. G. MacFarlane's memoir.

$$
\begin{aligned}
& r_{p}=\frac{X^{2} \alpha^{2}-\left(X+Z_{0} \alpha\right)^{2}+j 2 X \alpha\left(X+Z_{0} \alpha\right)}{\left(X+Z_{0} \alpha\right)^{2}+X^{2} \alpha^{2}} \\
& \operatorname{tg} \phi_{p}=\frac{2 X \alpha\left(X+Z_{0} \alpha\right)}{X^{2} \alpha^{2}-\left(X+Z_{0} \alpha\right)^{2}} ; \operatorname{tg} \frac{\phi_{p}}{2}=\frac{X+Z_{0} \alpha}{X \alpha}
\end{aligned}
$$

Moreover:

$$
r_{n}=\frac{\left(\alpha^{2}-1\right)+j 2 \alpha}{1+\alpha^{2}} ; \operatorname{tg} \phi_{n}=\frac{2 \alpha}{\alpha^{2}-1} ; \operatorname{tg} \frac{\phi_{n}}{2}=-\alpha
$$

Since the circuits with distributed constants of figure 3 are purely reactive, the moduli either of $r_{p}$ or of $r_{n}$ are unity.

It is easy to see that in order to achieve a converter of type $(\hat{A})$, entirely equivalent to a $\lambda / 4$ plate, then one must have

$$
\phi_{p}-\phi_{n}= \pm \frac{\pi}{2}
$$

To achieve type (B), which is equivalent to a $\lambda / 2$ plate, then one must, instead, have

$$
\phi_{p}-\phi_{n}= \pm \pi .
$$

$\left(\mathrm{A}^{\prime \prime}\right)$ - In case $(\mathrm{A})$, it is necessary that

$$
\phi_{p}-\phi_{n}= \pm \frac{\pi}{2} ; \frac{\phi_{p}}{2}=\frac{\phi_{n}}{2} \pm \frac{\pi}{4}
$$



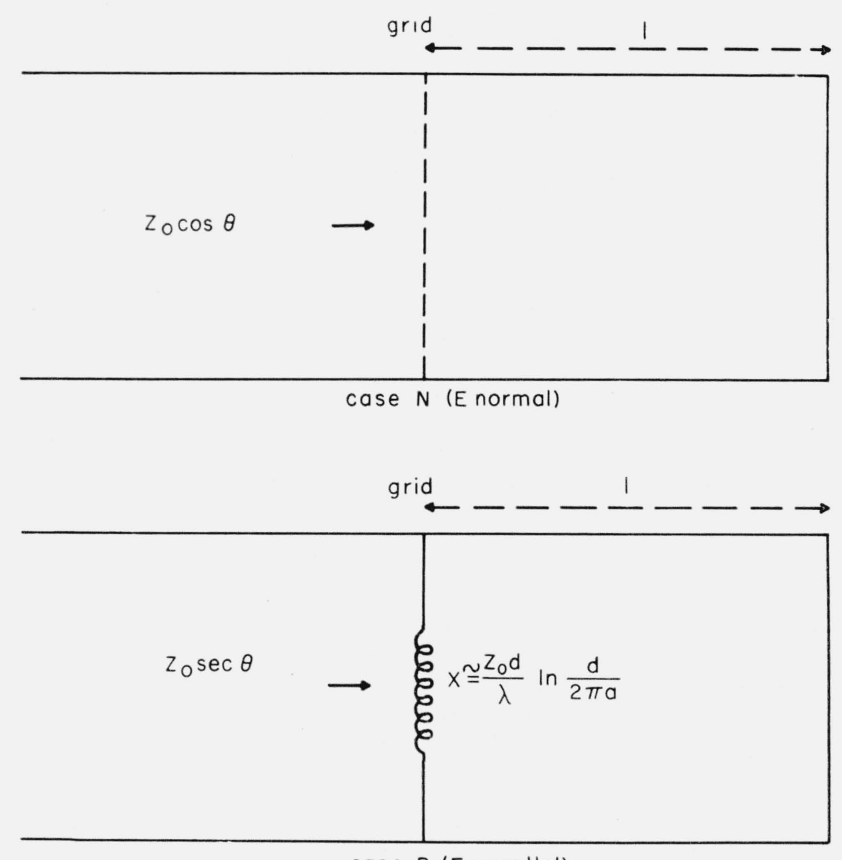

case $P$ (E parallel)

Figure 3. Operation of a wire grid in front of a plane reflector.

Wave has an electrical vector $E$ parallel $(P)$ or normal $(N)$ to the wires.

Therefore, taking the tangent,

$$
\frac{X+Z_{0} \alpha}{X \alpha}=\frac{-\alpha \pm 1}{1 \pm \alpha}
$$

Eliminating solution with the + sign, which leads to a complex root $\alpha$ when $X<Z_{0}$, one obtains

$$
\alpha=\frac{Z_{0} \pm \sqrt{Z_{0}^{2}+4 X\left(Z_{0}-X\right)}}{2\left(Z_{0}-X\right)} .
$$

Putting $Z_{0}=376.5$ ohms, $X=143$ ohms (for the particular grid in question) one finds roots $\alpha_{1}$, and $\alpha_{2}$. Hence, the corresponding distances are $l_{1}=$ $\left(5.56+\mathrm{n} \lambda_{0} / 2\right) \mathrm{mm} ; l_{2}=\left(14.43+\mathrm{n} \lambda_{0} / 2\right) \mathrm{mm}$ with $n$ being some integer.

$\left(\mathrm{B}^{\prime \prime}\right)$ - In case (B), $\phi_{n}=\phi_{\pi} \pm \pi$. Hence, taking the tangent

$$
\frac{2 \frac{X+Z_{0} \alpha}{X \alpha}}{1-\left(\frac{X+Z_{0} \alpha}{X \alpha}\right)^{2}}=\frac{2 \alpha}{\alpha^{2}-1}
$$

or

$$
\frac{2 t g \frac{\phi_{p}}{2}}{1-\operatorname{tg}^{2} \frac{\phi_{p}}{2}}=-\frac{2 \alpha}{1-\alpha^{2}},
$$

which leads to the solution

$$
\frac{X+Z_{0} \alpha}{X \alpha}=\left\{\begin{array}{l}
-\alpha \\
1 / \alpha
\end{array}\right.
$$

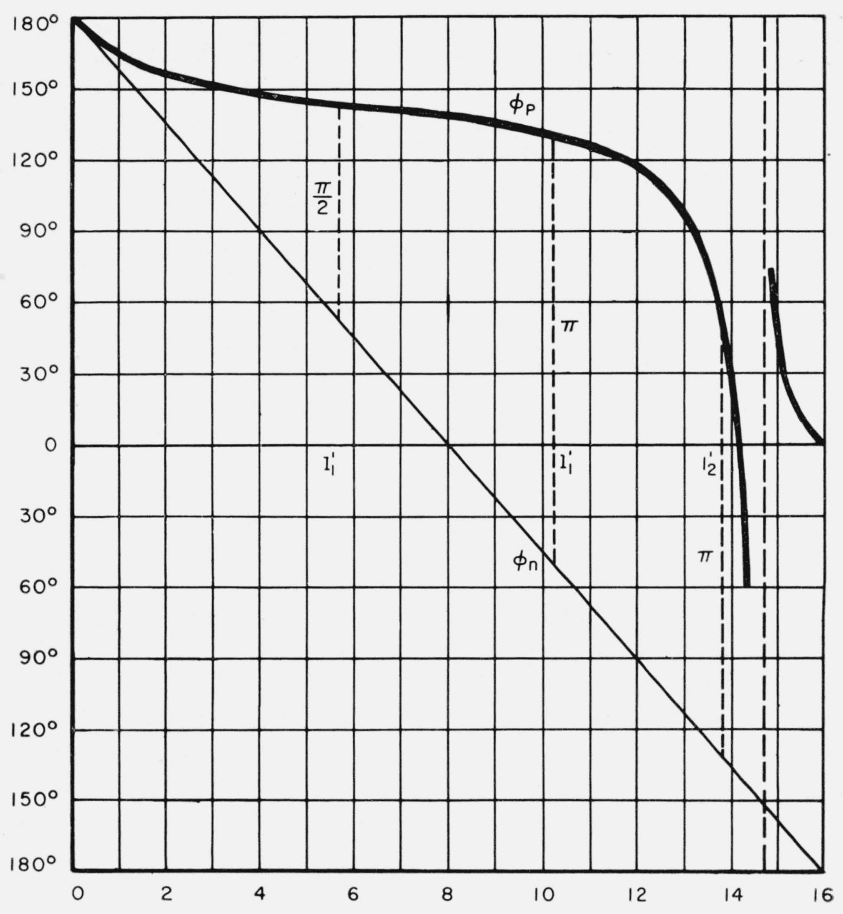

FiguRE 4. Graph for determining distance 1 between grid and reflector for which the difference between $\phi_{p}$ and $\phi_{n}$ is given an assigned value, e.g., 90 $($ Case $A)$ and $180^{\circ}$ (Case B).

Discarding the second alternative, which is physically impossible because it implies that $\alpha=0$, and $l=0$ (in this case $X$ would be short circuited), the following results are obtained after taking the first alternative,

$$
l_{1}^{\prime}=\left(10.19+\frac{n \lambda_{0}}{2}\right) m m ; l_{2}^{\prime}=\left(13.8+\frac{n \lambda_{7}}{2}\right) m m
$$

with $n$ equal to some integer.

The solution of problems A, B can also be found graphically by determining which abscissas correspond to a difference of $90^{\circ}$ between the $\phi_{p}$ and $\phi_{n}$ curves (fig. 4). Obviously, the graph is valid for the value of $X$ which we chose (143 ohms).

It is important to note that reflection polarizers make it possible to attain close to 100 percent conversion; in "transparent" converters, a substantial part of the incident energy is reflected instead.

\section{Tests With Wire Grids Placed in Front of Plane Reflectors-Experimental Setup}

Case A: Conversion of polarization from circular to linear and vice versa (wire grid at $45^{\circ}$ relative to the incident electric vector).

For operation in a noncritical region, where $\phi_{p}-\phi_{n}$ does not vary too rapidly with l, between two possible solutions (cf preceding sec. $\left(\mathrm{A}^{\prime \prime}\right)$ ), that solution is preferred (cf fig. 4) for which $l=5.56$ $+n \lambda_{0} / 2$. Further, if $n=2$, and the test is made with $\lambda_{0}=32 \mathrm{~mm}$, then $\mathrm{l}=37.56 \mathrm{~mm}-37 \div 38 \mathrm{~mm}$. The lack of precision inherent either in the construction 


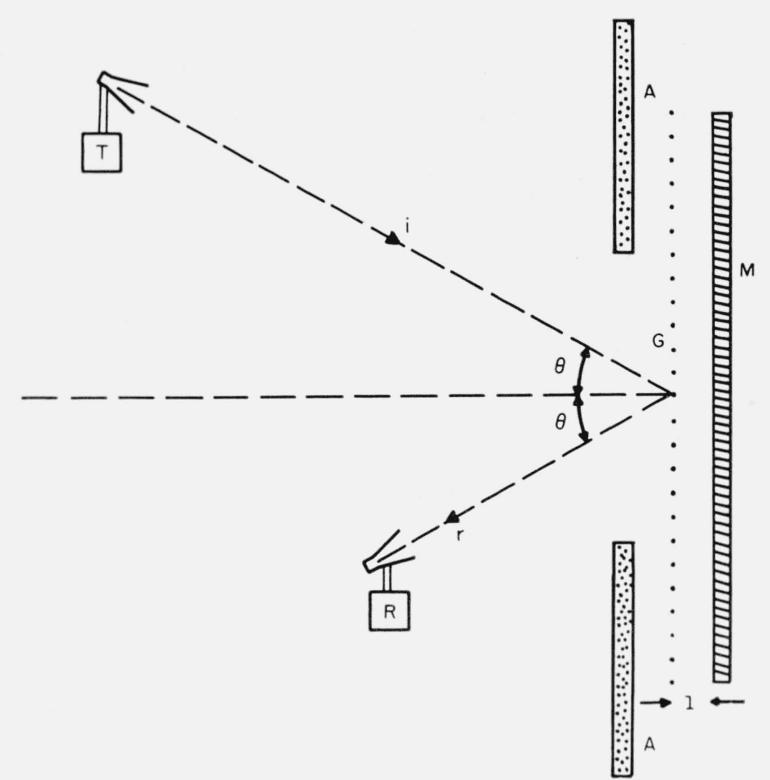

Figure 5. Schematic of the tests described in section 4.

$T=$ signal generator and transmitting horn: $\lambda_{0}=32 \mathrm{~mm}$ $R=$ receiving horn and receiver $A=$ absorbing panels $i=$ incident ray $l=$ variable distance

$G=$ parallel wire grid; vertical wires; radius $a=0.5 \mathrm{~mm}$; distance $d=10$ $\mathrm{mm}$; reactance $X=143 \mathrm{ohms}$ $M=$ plane metal reflector $r=$ reflected ray, with polarization different from incident ray

of the grid or in the spacing of the grid from the plane reflector does not, in fact, assure a precision better than $\pm 0.25 \mathrm{~mm}$ in $d$ (distance between wires) or $\pm 0.5 \mathrm{~mm}$ in 1 (distance between grid and reflector). The experimental setup is that shown schematically in figure 5. As the transmitting and receiving horns had a gain of only $16 \mathrm{db}$, it was necessary to install them at a distance of only a few meters from the grid and, furthermore, to screen by means of absorbing panels $(\sim 20 \mathrm{db})$ the frame of the grid and the peripherical part of it, so as to avoid disturbing effect. The distance between the horns and the grid was, however, sufficiently large to make the first Fresnel area larger than the free portion of the grid.

Operating with a sufficiently small angle $\theta$, which does not in our experience give sensitive corrections for normal incidence $\left(\theta<10^{\circ}\right)$, and with vertical wires, the following average results, illustrated under figure 6 ( $\mathrm{db}$ versus direction), were observed:

(1) Incident electric vector inclined $45^{\circ}$ to the right relative to the sense of propagation: emerging polarization approximately elliptical, with a difference of $\sim 2 \mathrm{db}$ between the two axes.

(2) Electric vector inclined $45^{\circ}$ to the left: same results as above.

The fact that the resulting polarization does not appear exactly circular but slightly elliptical can be amply justified by the imperfection of the experimental adjustments.

Case B: Rotation by $90^{\circ}$ of the electric vector of a linearly polarized incident wave, whose electric vector is at $45^{\circ}$ relative to the wires; or conversion from right to left circular and vice versa.

With the same setup as in case A, items 1 and 2, but giving 1 a value $\mathrm{l}=10,2 \mathrm{~mm}$ (cf. sec. $4,\left(\mathrm{~B}^{\prime \prime}\right)$ ), average results obtained proved that in the reflected
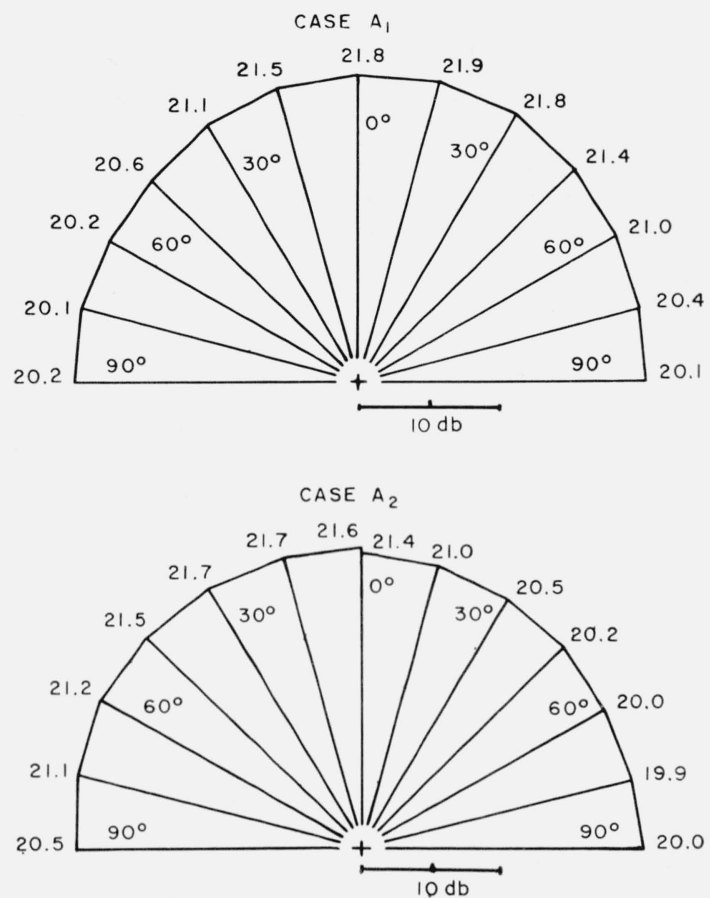

FiguRE 6. Graphs (db versus direction) illustrating the experiments of polarization conversion (from linear to circular) referred to in section 4, Cases $A_{1}$ and $A_{2}$ (incident electric vector inclined respectively $45^{\circ}$ to the right or to the left.)
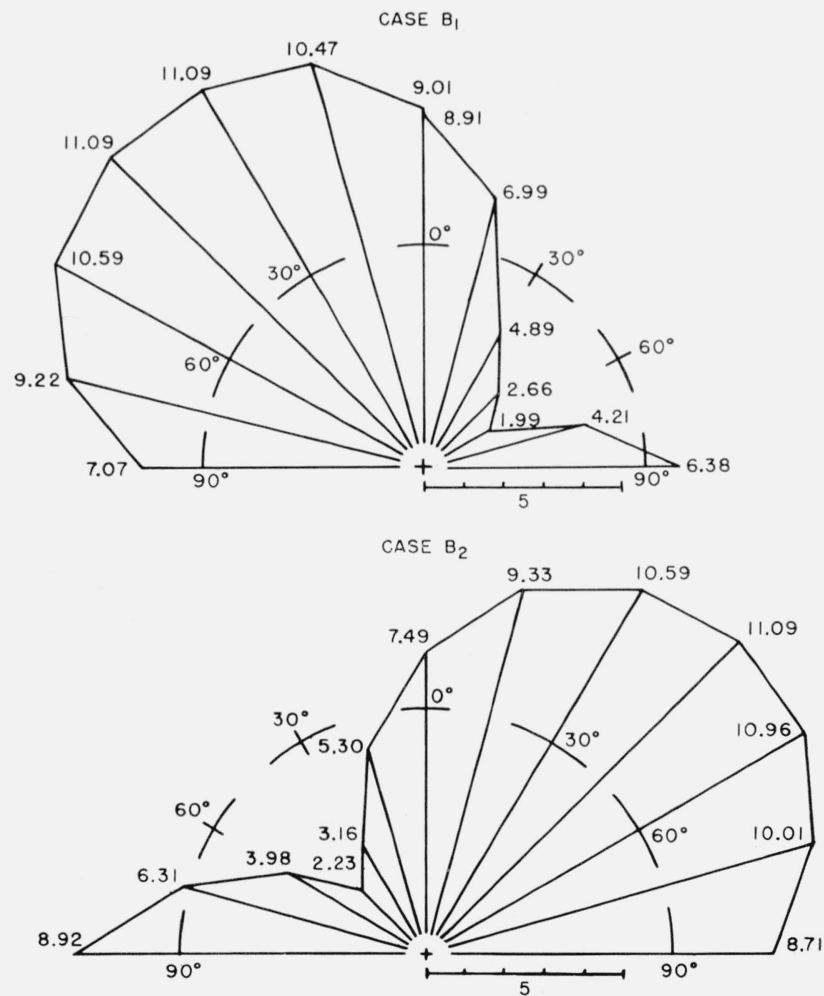

Figure 7. Graphs (field intensity versus direction) illustrating the polarization conversion experiments (rotation by $90^{\circ}$ ) referred to in section 4 case $B ; \mathrm{B}_{1}$ and $\mathrm{B}_{2}$ refer respectively to the cases that the incident electric vector is rotated by $45^{\circ}$ to the right or to the left. 
wave the conversion was almost complete. The reflected wave was elliptically polarized and the major axis was rotated by $90^{\circ}$ relative to the incident electric vector. The rate between major and minor axis was of $14 \div 15 \mathrm{db}$ (see fig. 7, field intensity versus direction, keeping in mind that a linear polarization would give a cosine law).

Beside the mechanical inexactitudes, one more cause for imprecision is that formula (1) assumes a wire grid of infinite extent and a plane incident wave; conditions which are not met in the above experiments. Furthermore, the radiation pattern of the receiving horn, which is rotated to explore the polarization of the reflected field, is not symmetrical relative to the horn-grid center line of sight. However, from the above referred data it appears that, notwithstanding the rough experimental setup, the polarization properties of reflection converters $\mathrm{A}$ and $\mathrm{B}$ have been demonstrated.

\section{Corner Reflectors With Wire Grids in Front of Them}

Using the principle involved in, but disregarding the lack of precision in the application of equations (1) and (2) to a grid of extremely reduced dimensions, one may apply the technique of section 4 (discussed experimentally in sec. 5), to the case of dihedral or trihedral reflectors. In this case the reflected ray always assumes a direction equal to, but in a sense opposite to that of the incident ray. This well known principle from optics is used to construct radar passive reflectors known as "corner reflectors."

We recalled in section 2 that these reflectors, when built as trihedrals - in practice most useful for naviga-

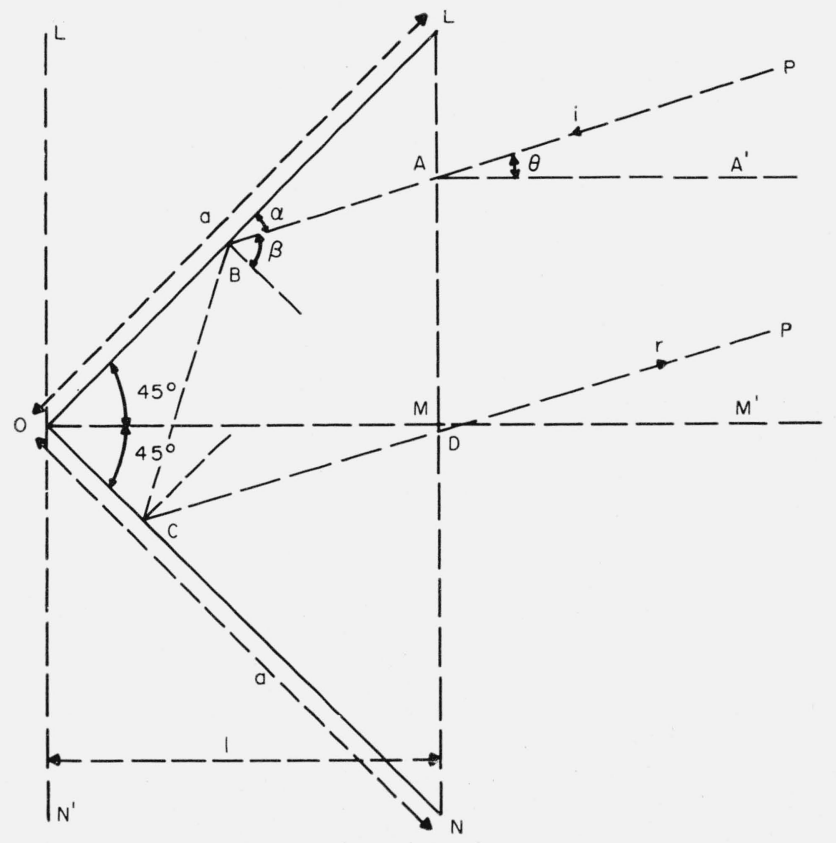

FiguRE 8. Cross section of a dihedral corner reflector; ABCD $=21 / \cos \theta$. tion - were nonvisible with radar with circular polarization ("antirain") since for every reflection the circular polarization inverts its direction, whereas one or three reflections (in the case of plane or trihedral reflectors) result in radarvisibility.

A polarization converter of type B having the property of transforming horizontal polarization to vertical and vice versa as well as inverting the direction of circular polarization, should become fully radarvisible with circular but invisible with linear (cf. sec. 2, parts $\mathrm{A}$ and $\mathrm{B}$ ). With type $\mathrm{A}$ one may have radarvisibility with linear (horizontal or vertical) or with circular polarization, with a loss in every case of $3 \mathrm{db}$. However, to apply the technique of the preceding section to the case of corner reflectors, it is helpiul to prove that the "internal path" does not depend upon the point of incidence of the ray on the "aperture" of the corner reflector.

One may take as an example the right dihedral reflector shown in cross section in figure 8. From a simple geometrical deduction, it can be shown that the incident ray $i$ and the reflected ray $r$ are parallel if the angle at $\mathrm{O}$ is a right angle. Also, the "internal path" $P_{i}=\mathrm{ABCD}$ does not depend upon the incidence point A on the "aperture" LN but only on the inclination $\theta$ of the incident ray relative to the "axis" OM of the dihedral.

It is just as easily deduced that the "internal path" $P_{i}$ is

$$
\begin{aligned}
& P_{i}=\mathrm{ABCD}=2 \frac{a}{\cos \alpha+\cos \beta} \\
& =2 \frac{a}{\cos \alpha+\sin \alpha}=2 \frac{1}{\cos \theta}=\frac{2 a}{\sqrt{2} \cos \theta}
\end{aligned}
$$

It all comes to this: after internal reflection there is formed within the "aperture" LN of the corner reflector a reflecting surface $L^{\prime} \mathrm{N}^{\prime}$ at a distance $\mathrm{l}=\mathrm{OM}$.

In the case of a trihedral, the demonstration becomes more complicated but the result is altogether analogous :

$$
P_{i}=2 \frac{a}{\cos \beta+\cos \gamma+\cos \alpha}=2 \frac{1}{\cos \theta}=2 \frac{a}{\sqrt{3} \cos \theta}
$$

For a small $\theta$, e.g., $\theta<10^{\circ}$, one may place $P_{i}=2 \mathrm{l}=\frac{2 a}{\sqrt{3}}$ to operate a converter of polarization of, say, type B, with a trihedral reflector, using a grid of cylindrical wire (radius $0.5 \mathrm{~mm}$ ) and spaced $10 \mathrm{~mm}$ as previously tested ( $X=143$ ohms) and using a small $\theta$ (e.g., $<10^{\circ}$ ), it would be sufficient (cf. sec. 3 and 4 , case B) to put

$$
P=2 l=\left(10.2+\frac{n \lambda_{0}}{2}\right) \mathrm{mm} .
$$

In the corner reflector used in the tests (see fig. 9) $n=20$, and correspondingly $\left(\lambda_{0}=32 \mathrm{~mm}\right) \mathrm{l} \simeq 165 \mathrm{~mm}$.

It was found, as anticipated, that the limitations of the grid, and the fact that its edges were short circuited from the reflector plates, precluded obtain- 


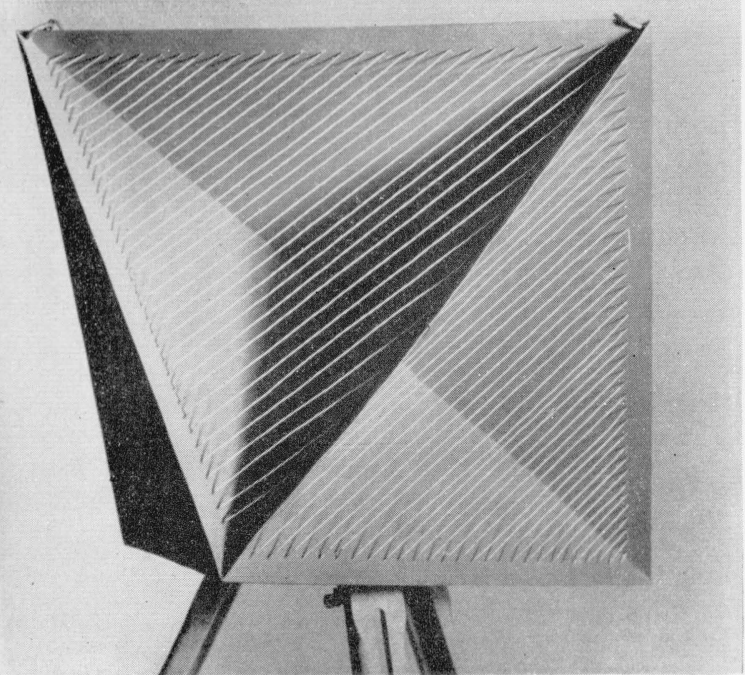

FiguRE 9. Corner reflector equipped with parallel wire grid.

ing results such as those which could have been rigorously expected in accord with equations (1) and (2), from simple deductions from optical geometry, above all when the incident polarization is parallel to a plane of the reflector. In particular, it becomes, thus, difficult to convert correctly horizontal into vertical polarization. The converted polarization is elliptical, the major axis, however, is always rotated by $90^{\circ}$ respect to the incident electric vector.

The average results obtained are to be found in following tabulated summary:

Tests with a trihedral reflector

$\left(\lambda_{0}=32 \mathrm{~mm}\right)$

\begin{tabular}{|c|c|c|c|c|}
\hline & \multicolumn{2}{|c|}{$\begin{array}{l}\text { Reflected signal } \\
\text { without depolariz- } \\
\text { ing grid }\end{array}$} & \multicolumn{2}{|c|}{$\begin{array}{l}\text { Reflected signal } \\
\text { with depolarizing } \\
\text { grid }\end{array}$} \\
\hline & $\begin{array}{l}\text { Horiz. } \\
\text { polariz. }\end{array}$ & $\begin{array}{c}\text { Vert. } \\
\text { polariz. }\end{array}$ & $\begin{array}{c}\text { Horiz. } \\
\text { polariz. }\end{array}$ & $\begin{array}{c}\text { Vert. } \\
\text { polariz. }\end{array}$ \\
\hline $\begin{array}{l}\text { (1) Transmitted } \\
\text { polarization: } \\
\text { horizontal_-. } \\
\text { (2) Transmitted } \\
\text { polarization: } \\
\text { vertical_... }\end{array}$ & $\begin{array}{l}\sim 0 \mathrm{db} \\
<-20 \mathrm{db}\end{array}$ & $\begin{array}{l}<-20 \mathrm{db} \\
\sim 0 \mathrm{db}\end{array}$ & $\begin{array}{l}-4 \mathrm{db} \\
\sim 0 \mathrm{db}\end{array}$ & $\begin{array}{l}\sim 0 \mathrm{db} \\
\sim-9 \mathrm{db}\end{array}$ \\
\hline
\end{tabular}

\section{Conclusion}

The considerations just developed and the ensuing tests allow us to make the following conclusion:

An efficient setup intended to convert "by reflection" (A) linear polarization to circular, or (B) hori- zontal to vertical (or right to left circular) and vice versa, can be attained easily by placing a properly designed wire grid in front of a plane reflector.

One may reach a very high degree of conversion (almost 100 percent) compared to what can ordinarily be done with a "transparent" converter. Moreover, the effect of the converter can be estimated from simple calculations as long as the wire grid is of such dimensions to allow it to be dealt with as indefinite in extent. Whenever a corner and, in particular, a trihedral reflector is used rather than a plane one, then theoretical precision based on assumptions of the preceding section and considerations from the geometry of optics, does not come to any close agreement with experimental results. It is particularly difficult to achieve a type $B$ converter. Type $\dot{A}$ can be approximated very easily within acceptable tolerances and applied to the practical purpose of obtaining with radar for exchangeable polarization from linear (horizontal or vertical) to circular ("antirain"), echoes with loss not over $3 \mathrm{db}$ from trihedral reflectors equipped with wire grid at $45^{\circ}$.

If in the future, as is probable, the use of radar is extended to produce exchangeable polarization, then the use of type A reflectors could be suitably introduced. As for type B polarization converters "by reflection," it may be of interest to point out that such converters change the right to left-circular polarization and vice versa, and consequently invert as well the associated em angular momentum [Carrara, 1949 ; Carrara et al., 1955] in the same way as a conducting plate, normal to the direction of propagation of a plane wave, does for the linear momentum.

Thanks are due in a very special way to the degree candidate Lorenzo Mirabile, who took charge of conducting the tests.

\section{References}

Carrara, N., Coppia e momento angolare della radiazione, Il Nuovo Cimento 4, No. 1, 50-56 (January 1949).

Carrara, N., T. Fazzini, L. Ronchi, and G. Toraldo di Francia, Sul momento di rotazione del campo elettromagnetico, Alta frequenza 24, No. 2, 100-109 (April 1955).

Copeland, J. R., Radar target classification by polarization properties, Proc. I.R.E. 48, No. 7, 1290-1296 (July 1960).

Decker, M. T., Transmission and reflection by a parallel wire grid, J. Research NBS 63D (Radio Prop.), No. 1, 87-90 (July-August 1959).

MacFarlane, G. G., Surface impedance of an infinite parallelwire grid at oblique angles of incidence, J. Inst. Elec. Engrs. (London) 93, pt. III A, 1523 (1946).

Wait, J. R., Reflection at arbitrary incidence from a parallel wire grid, Appl. Sci. Research B4, No. 6, 393-400 (June $1954 a)$.

Wait, J. R., Reflection from a wire grid parallel to a conducting plane, Can. J. Phys. 32, 571-579 (Sept. 1954b).

(Paper 66D1-171) 